\title{
Folliculitis decalvans and orofacial granulomatosis
}

\author{
Kaja Męcińska-Jundziłt, Agnieszka Białecka, Urszula Adamska, Grażyna Kupś-Chmara, Aleksandra Grzanka, \\ Rafał Czajkowski
}

Clinic of Dermatology, Sexually Transmitted Diseases and Immunodermatology, Faculty of Medicine in Bydgoszcz, Nicolaus Copernicus University in Torun, Poland

Adv Dermatol Allergol 2018; XXXV (3): 317-319

DOI: https://doi.org/10.5114/pdia.2017.70261

Folliculitis decalvans (FD) is a rare and chronic inflammatory dermatosis which pathogenesis remains unclear. It is classified as primary neutrophilic cicatricial alopecia presented clinically with follicular pustules and papules, hemorrhagic crusts, erosions and scarring within scalp. Orofacial granulomatosis (OFG) is a recurrent or persistent orofacial swelling histopathologically presented with non-caseating granulomas. The etiology of the disease is not fully understood. There are hypotheses concerning pathogenesis of the disease including genetic, allergic, infectious and immunological factors. We report a case of a patient with FD and concomitant orofacial granulomatosis successfully treated with dapsone.

A 32-year-old man was admitted to the Department of Dermatology to diagnose follicular pustules, erosions, yellow scales, crusts with scarred areas and focal hair loss within the scalp, especially within vertex and accompanying persistent lip swelling with normal appearance of the tongue and no oral lesions (Figure 1). Moreover, we observed erythrosquamous lesions and papules on the lateral surfaces of the trunk, within groins and armpits. The lesions on the scalp occurred in childhood and the lip swelling persisted for 3 years. Except skin eruptions the patient did not report any other complaints. He was initially treated in another clinic for seborrheic dermatitis and acne inversa topically with antibiotics, glucocorticosteroids, tacrolimus and orally with antibiotics (doxycycline), antihistamines with temporary improvement. The patient suffered from post-traumatic epilepsy and was treated with carbamazepine. He had a craniotomy due to head injury (concussions and subdural hematoma of the left temporo-parietal brain region, basilar skull fracture and squamous part of the right temporal bone fracture) during the traffic accident 20 years earlier. During the hospitalization routine laboratory tests showed an elevated C-reactive protein level and erythrocyturia which occurred to be irrelevant (the patient was consulted by the urologist). Swabs from erosions and pustules were collected and two types of bacteria were cultured - Staphylococcus aureus and Streptococcus agalactiae. To exclude concomitant systemic diseases, we performed additional examinations. There were no abnormalities in abdominal ultrasonography or chest X-ray. Antinuclear antibodies, lupus anticoagulant, cardiolipin antibodies (IgG and $\lg M), \beta_{2}$-glycoprotein 1 antibodies (IgG and $\lg M$ ), anti-Borrelia spp. antibodies tests (IgG and IgM), concentration of $\mathrm{C} 1$ esterase inhibitor and Quantiferon-TB Gold test in patient's serum were negative or within normal
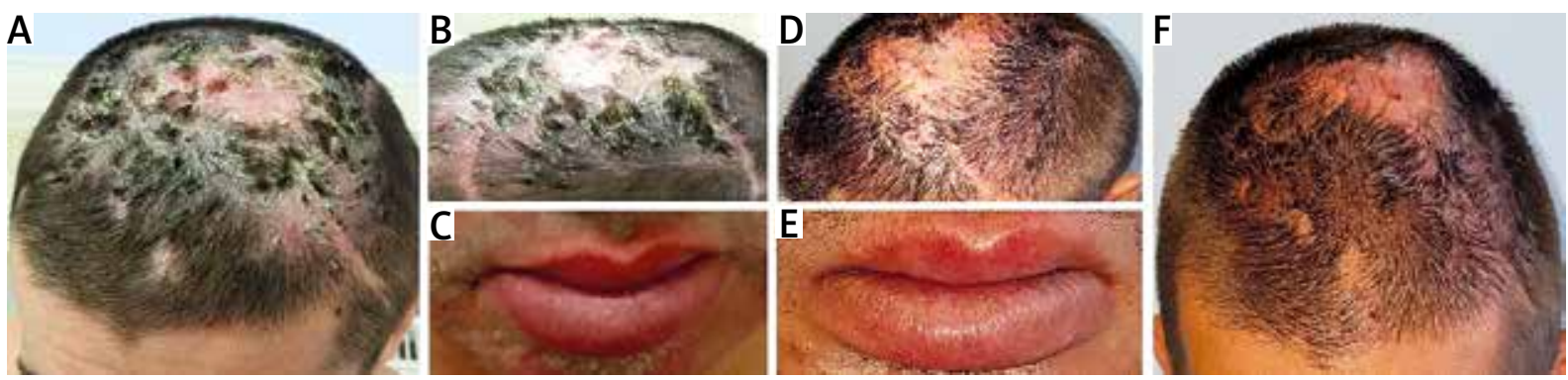

Figure 1. A, B - Lesions within scalp, C-persistent lip swelling, D-F - improvement of skin lesions in the course of treatment with dapsone

Address for correspondence: Kaja Męcińska-Jundziłł MD, Clinic of Dermatology, Sexually Transmitted Diseases and Immunodermatology, Faculty of Medicine, Nicolaus Copernicus University, 9 Sklodowskiej-Curie St, 85-094 Bydgoszcz, Poland, phone: +48 602404829 , e-mail: kaja_cm@wp.pl

Received: 11.06.2017, accepted: 12.08.2017. 

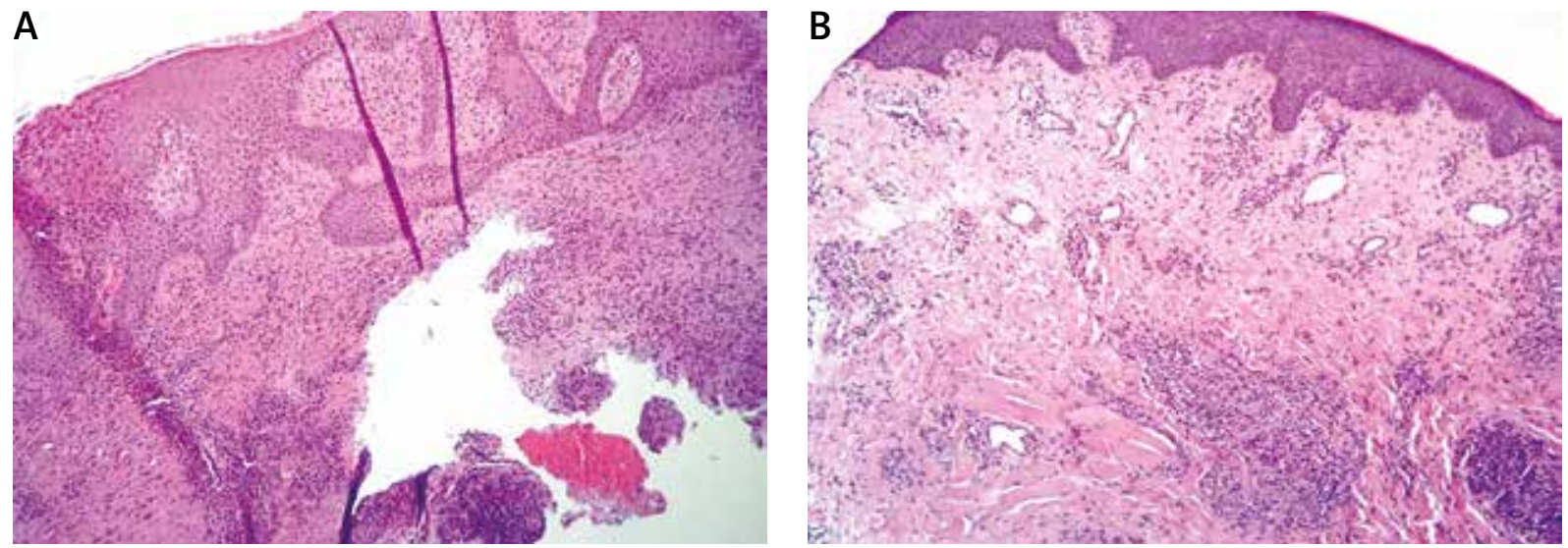

Figure 2. $\mathrm{H}+\mathrm{E}$ staining. A - Histopathological image of the scalp lesion with epidermal pustules, dermis infiltrations of lymphocytes, plasmocytes and neutrophils, absence of hair follicles, B - histopathological image of the lip showing noncaseating granulomas

values. Patch tests with 30 allergens were negative in every disc in 48 and $72 \mathrm{~h}$ tests. The patient was consulted neurologically to exclude paralysis of the facial nerve, which is one of the symptoms of Melkersson-Rosenthal syndrome. The neurological examination revealed slight features of the central nervous system failure which most likely occurred due to the head injury, there were no signs of facial palsy. Histopathology of the scalp skin lesion revealed absence of hair follicles, epidermal pustules, dermis infiltrations of lymphocytes, plasmocytes and neutrophils, stains for fungi infection were negative (Figure $2 \mathrm{~A}$ ). Histopathology of the lip showed non-caseating granulomatous inflammation (Figure 2 B). Indirect and direct immunofluorescence were negative. Based on the patient history, physical examination, histopathology and laboratory analysis we identified FD and orofacial granulomatosis. Treatment with dapsone of $100 \mathrm{mg} /$ day combined with topical corticosteroids (betamethasone, clobetasol) and antibiotics (gentamicin, detreomicin) was started and led to significant improvement.

Folliculitis decalvans mostly occurs in young and middle-aged adults with a slight predilection to men [1]. Its pathogenesis is not clear. Familial cases of the disease were reported [2, 3]. Moreover it seems that the disease is a hypersensitivity reaction to Staphylococcus aureus follicle infection as in majority of patients the bacteria are isolated from lesions as it was in our case [3,4]. There are single reported cases where the lesions occurred after scalp injuries what may be relevant as our patient had a head injury and craniotomy in the childhood [5, 6]. In majority of cases, FD is clinically presented with pustules, tufted hairs, alopecic patches, erosions and crusts especially within the vertex, what is consistent with our case $[1,3]$. Histopathological examination of early lesions reveal papular lesions, perifollicular infiltration mainly consisted of neutrophils lymphocytes and plasma cells and early scarring, in long lasting lesions the infiltration extend deeper into the dermis [1, 3]. The treatment of FD is a challenge because there is no single therapy which leads to long-term remission. In reported cases various therapies were conducted for example systemic antibiotics (especially rifampicin with clindamycin, doxycycline) [3, 7], isotretinoin [8], dapsone [9], frequently in combination with topical corticosteroids and antibiotics. What is important, regular monitoring of the patient is necessary as there are reports on squamous cell carcinoma arising within FD [10]. The term of orofacial granulomatosis was introduced in 1985 by Wiesenfeld et al. to describe idiopathic orofacial edema with granulomatous inflammation in the absence of systemic diseases, what is consistent with our case [11]. Orofacial granulomatosis encompasses other known granulomatous diseases such as Melkersson-Rosenthal syndrome which consists of three symptoms: persistent orofacial edema, recurrent facial paralysis and fissured tongue and Miescher's cheilitis, which refers to a condition when lip swelling is an isolated symptom or granulomatous cheilitis [12, 13]. Oral granulomas may occur in systemic conditions such as Crohn's disease, sarcoidosis or tuberculosis so they must be excluded during diagnostic procedures [12]. The etiology of the disease is not fully understood. There are hypotheses concerning pathogenesis of the disease including genetic, allergic (food allergy, allergy to dental materials), infectious (especially tuberculosis) and immunological factors [12]. In our patient we observed erythrosquamous lesions and papules on the lateral surfaces of the trunk, within groins and armpits, what suggested concomitant contact dermatitis although patch tests were negative, further allergy diagnostics would be required. Because etiology of the disease is not clear, the treatment is difficult. When allergy is detected, avoidance of allergens may lead to improvement. In most cases, oral and intralesional corticosteroids were used, moreover there are some reports of topical tacrolimus, clofazimine, thalidomide, dapsone, and infliximab treatment [12-15]. In our case, diagnosis and treatment of two 
coexisting diseases which etiologies are not fully understood and require multidirectional diagnostic procedures with no single therapies providing satisfying effects was a great challenge. Fortunately, treatment with dapsone combined with topical ointments led to significant improvement.

\section{Acknowledgments}

Kaja Męcińska-Jundziłł and Agnieszka Białecka are the first authors.

\section{Conflict of interest}

The authors declare no conflict of interest.

\section{References}

1. Otberg N, Kang H, Alzolibani AA, Shapiro J. Folliculitis decalvans. Dermatol Ther 2008; 21: 238-44.

2. Jaiswal AK, Vaishampayan S, Walia NS, Verma R. Folliculitis decalvans in a family. Indian J Dermatol Venereol Leprol 2000; 66: 216-7.

3. Vańó-Galván S, Molina-Ruiz AM, Fernández-Crehuet P, et al. Folliculitis decalvans: a multicentre review of 82 patients. J Eur Acad Dermatol Venereol 2015; 29: 1750-7.

4. Powell JJ, Dawber RP, Gatter K. Folliculitis decalvans including tufted folliculitis: clinical, histological and therapeutic findings. Br J Dermatol 1999; 140: 328-33.

5. Fernandes JC, Correia TM, Azevedo F, Mesquita-Guimarães J. Tufted hair folliculitis after scalp injury. Cutis 2001; 67: 243-5.

6. Otberg N, Wu WY, Kang H, et al. Folliculitis decalvans developing 20 years after hair restoration surgery in punch grafts: case report. Dermatol Surg 2009; 35: 1852-6.

7. Bunagan MJK, Banka N, Shapiro J. Retrospective review of folliculitis decalvans in 23 patients with course and treatment analysis of long-standing cases. J Cutan Med Surg 2015; 19: 45-9.

8. Tietze JK, Heppt MV, von Preußen A, et al. Oral isotretinoin as the most effective treatment in folliculitis decalvans: a retrospective comparison of different treatment regimens in 28 patients. J Eur Acad Dermatol Venereol 2015; 29: 1816-21.

9. Paquet P, Piérard GE. Dapsone treatment of folliculitis decalvans. Ann Dermatol Venereol 2004; 131: 195-7.

10. Yip L, Ryan A, Sinclair R. Squamous cell carcinoma arising within folliculitis decalvans. Br J Dermatol 2008; 159: 481-2.

11. Wiesenfeld D, Ferguson MM, Mitchell DN, et al. Oro-facial granulomatosis: a clinical and pathological analysis. Q J Med 1985; 54: 101-13.

12. Grave B, McCullough M, Wiesenfeld D. Orofacial granulomatosis - a 20-year review. Oral Dis 2009; 15: 46-51.

13. Miest R, Bruce A, Rogers RS. Orofacial granulomatosis. Clin Dermatol 2016; 34: 505-13.

14. Lynde CB, Bruce AJ, Orvidas LJ, et al. Cheilitis granulomatosa treated with intralesional corticosteroids and anti-inflammatory agents. J Am Acad Dermatol 2011; 65: e101-2.

15. Al Johani KA, Moles DR, Hodgson TA, et al. Orofacial granulomatosis: clinical features and long-term outcome of therapy. J Am Acad Dermatol 2010; 62: 611-20. 